\title{
AUTHOR INDEX Volume 20
}

Abbas, A., see Raval, P.

20 (2017) 1750018

Abdullah, M., see Haryono, I. R.

20 (2017) 1750012

Ahmad, M., see Tayyem, M.

20 (2017) 1750009

Akbari, K., see Salunke, A. A.

20 (2017) 1772001

Ananzah, M., see Tayyem, M.

Arias, A. A., see Romero, E. C.

20 (2017) 1750009

20 (2017) 1750022

Asadi, M. and Roshanzamir, S., Electrodiagnostic Criteria for Diagnosing Carpal Tunnel Syndrome Need to be Revised in Hypothyroid Patients

20 (2017) 1750014

Ashtiani, M. N. and Azghani, M.-R., Predictive Models for Estimation of the Human Stance Equilibrium Parameters Using Inverse Dynamics and Response Surface Method

Azghani, M.-R., see Ashtiani, M. N.

Bhalodiya, P., see Salunke, A. A.

Candel, R. P., see Romero, E. C.

Chakravarty, R. D., see Harikesavan, K.

Chappell, I., Lee, P., Mciff, T. E., Toby, E. B. and Fischer, K. J., Effects of Scapholunate Ligament Injury and Surgical Repair on Wrist Cartilage T2 Relaxation Time

Chen, Y., see Salunke, A. A.

Choisne, J., Travert, C., Valiadis, J.-M., Follet, H. and Skalli, W., A New Method to

Determine Volumetric Bone Mineral Density from Bi-Planar Dual Energy

Radiographs Using a Finite Element Model: An Ex-Vivo Study

Davis, E. T., see Sajid, S.

Dimitriadis, Z., Fragkakis, V., Kapreli, E., Strimpakos, N. and Petta, G., Does Motivation Affect the Performance in Clinical Tests of Endurance of Deep Neck Flexors?

Dwivedi, C., see Salunke, A. A.

Eapen, C., see Sahasrabudhe, A.

Elhadid, F., see Tayyem, M.

Evangelou, A. M., see Oikonomidis, S. A.

Evans, T. J., Frisch, N. B., Gibson, G., Mott, M. P. and Parsons III, T. W., Validation of a Rat Model for Analyzing MicroRNA in Chondrosarcoma

Fischer, K. J., see Chappell, I.

Follet, H., see Choisne, J.

Fragkakis, V., see Dimitriadis, Z.

Frisch, N. B., see Evans, T. J.

Garikayi, T., Van den Heever, D. and Matope, S., Investigating the Effects of Passive Mechanical Ankle on Unilateral Osteomyoplastic Transtibial Amputees

20 (2017) 1750016

20 (2017) 1750016

20 (2017) 1772001

20 (2017) 1750022

20 (2017) 1750017

20 (2017) 1750006

20 (2017) 1772001

20 (2017) 1750003

20 (2017) 1750001

20 (2017) 1750019

20 (2017) 1772001

20 (2017) 1750023

20 (2017) 1750009

20 (2017) 1750008

20 (2017) 1750021

20 (2017) 1750006

20 (2017) 1750003

20 (2017) 1750019

20 (2017) 1750021

20 (2017) 1750015 
Gibson, G., see Evans, T. J.

Girish, N., Iqbal, R. and Khanzode, V., Lifting Capacity Among Indian Manual Materials Handlers Using Progressive Isoinertial Lifting Evaluation

Gómez, S. G., see Romero, E. C.

Gonzalez, J. G., see Romero, E. C.

Haddad, R., see Tayyem, M.

Hamdan, M., see Tayyem, M.

Harikesavan, K., Chakravarty, R. D. and Maiya, A. G., Effects of Early Rehabilitation Protocol Post High Flex Cruciate Retaining Total Knee Replacement on Range of Motion, Pain and Function: A Prospective Study

Haryono, I. R., Tulaar, A., Sudoyo, H., Purba, A., Abdullah, M., Jusman, S. W., Lubis, A. M. T. and Ibrahim Ilyas, E. I., Comparison of the Effects of Walking and Bench-Step Exercise on Osteocalcin and CTX-1 in Post-Menopausal Women with Osteopenia

Hasegawa, Y., see Oishi, Y.

Hirano, Y., see Oishi, Y.

Homayounee, K., see Sanaee, S.

Ibrahim Ilyas, E. I., see Haryono, I. R.

Iqbal, R., see Girish, N.

Jain, S., see Salunke, A. A.

Jusman, S. W., see Haryono, I. R.

Kanayama, Y., see Oishi, Y.

Kapreli, E., see Dimitriadis, Z.

Karkabounas, S. Ch., see Oikonomidis, S. A.

Khanzode, V., see Girish, N.

Kim, D.-H., see Moon, M.-S.

Kim, D.-H., see Moon, M.-S.

Kosugi, S., see Nomura, K.

Kuiper, J. H., see Sajid, S.

Kumari, M., see Midde, A. K.

Lee, P., see Chappell, I.

Lubis, A. M. T., see Haryono, I. R.

Maiya, A. G., see Harikesavan, K.

Matope, S., see Garikayi, T.

Mciff, T. E., see Chappell, I.

Midde, A. K., Panigrahi, M., Kumari, M. and Tedla, J. S., Normative Values for Cervical Lordosis: A Cross-Sectional Study

Mishra, S., see Salunke, A. A.

Mizoguchi, H., see Nomura, K.

Mohammadi, G., Prevalence of Low Back Pain and Associated Risk Factors Among High School Teachers in Kerman, Iran

Moon, M.-S., Kim, D.-H., Park, B.-K. and Yoon, M.-G., Bone Healing Patterns of Interlocked Intramedullary Nail-Fixated Femoral Shaft Fractures: Age-Matched Radiographic Presentation of Union Pattern

Moon, M.-S., Park, M.-S., Park, B.-K., Kim, D.-H. and Yoon, M.-G., Preoperative Nutritional Status of Hip Fracture Patients: A Pilot Study in 116 Patients

Mott, M. P., see Evans, T. J.

Nieto, D. P., see Romero, E. C.
20 (2017) 1750021

20 (2017) 1750004

20 (2017) 1750022

20 (2017) 1750022

20 (2017) 1750009

20 (2017) 1750009

20 (2017) 1750017

20 (2017) 1750012

20 (2017) 1750007

20 (2017) 1750007

20 (2017) 1750013

20 (2017) 1750012

20 (2017) 1750004

20 (2017) 1772001

20 (2017) 1750012

20 (2017) 1750007

20 (2017) 1750019

20 (2017) 1750008

20 (2017) 1750004

20 (2017) 1750002

20 (2017) 1750010

20 (2017) 1750011

20 (2017) 1750001

20 (2017) 1750020

20 (2017) 1750006

20 (2017) 1750012

20 (2017) 1750017

20 (2017) 1750015

20 (2017) 1750006

20 (2017) 1750020

20 (2017) 1772001

20 (2017) 1750011

20 (2017) 1750005

20 (2017) 1750010

20 (2017) 1750002

20 (2017) 1750021

20 (2017) 1750022 
Nomura, K., Yonezawa, T., Kosugi, S., Tanaka, Y., Takemura, H. and Mizoguchi, H., Three-Dimensional Posture Estimation of Foot Bones by Using Plantar Plate

Oikonomidis, A. S., see Oikonomidis, S. A.

Oikonomidis, S. A., Simos, Y. V., Toliopoulos, I. K., Verginadis, I. I., Oikonomidis, A. S., Ragos, V. N., Karkabounas, S. Ch., Evangelou, A. M. and Peschos, D., Oxidative Stress Incidence on the Severity of Knee Osteoarthritis

20 (2017) 1750011

20 (2017) 1750008

20 (2017) 1750008

Oishi, Y., Hirano, Y., Hasegawa, Y., Yamauchi, K., Kanayama, Y. and Ota, S., Prior Knee Osteoporosis Associating the 10-Year Clinical Outcome of Total Knee Arthroplasty for Rheumatoid Arthritis: A Retrospective Study

Ordoño, A. A., see Romero, E. C.

Ota, S., see Oishi, Y.

Panigrahi, M., see Midde, A. K.

Park, B.-K., see Moon, M.-S.

Park, B.-K., see Moon, M.-S.

Park, M.-S., see Moon, M.-S.

Parsons Iii, T. W., see Evans, T. J.

Pathak, S., see Salunke, A. A.

Peschos, D., see Oikonomidis, S. A.

Petta, G., see Dimitriadis, Z.

Purba, A., see Haryono, I. R.

Ragos, V. N., see Oikonomidis, S. A.

Raval, P. and Abbas, A., klebsiella Pneumoniae Causing Spondylodiscitis: An Unusual Cause of Back Pain in an Elderly Patient

Romero, E. C., Gómez, S. G., Candel, R. P., Ordoño, A. A., Arias, A. A., Gonzalez, J. G., Romero, R. C., Serrano, D. D. and Nieto, D. P., Unilateral versus Simultaneous Bilateral Percutaneous Hallux Valgus Surgery

Romero, R. C., see Romero, E. C.

Roshanzamir, S., see Sanaee, S.

Roshanzamir, S., see Asadi, M.

Sahasrabudhe, A., Eapen, C. and Zulfeequer, C. P., Comparison of Efficacy of Mechanical Intermittent and Kaltenborn Cervical Traction Mobilization with Belt in Radiating Neck Pain: A Randomized Clinical Trial

20 (2017) 1750007

20 (2017) 1750022

20 (2017) 1750007

20 (2017) 1750020

20 (2017) 1750002

20 (2017) 1750010

20 (2017) 1750002

20 (2017) 1750021

20 (2017) 1772001

20 (2017) 1750008

20 (2017) 1750019

20 (2017) 1750012

20 (2017) 1750008

20 (2017) 1750018

20 (2017) 1750022

20 (2017) 1750022

20 (2017) 1750013

20 (2017) 1750014

20 (2017) 1750023

Sajid, S., Kuiper, J. H. and Davis, E. T., Review of the Relationship between Knee Alignment and Fixed Flexion Deformity and How This Can Influence Total Knee Arthroplasty: A Retrospective Cohort Study

Salunke, A. A., Savale, A., Dwivedi, C., Pathak, S., Ughareja, P., Akbari, K., Jain, S., Bhalodiya, P., Mishra, S. and Chen, Y., Hoffa's Fracture with Irreducible Patellar

Dislocation: Approach to Avoid Complications in a Rare Injury

Samarah, O., see Tayyem, M.

Sanaee, S., Roshanzamir, S. and Homayounee, K., Efficacy of Long-Term Splinting in the Treatment of Severe Carpal Tunnel Syndrome

Savale, A., see Salunke, A. A.

Serrano, D. D., see Romero, E. C.

Simos, Y. V., see Oikonomidis, S. A.

Skalli, W., see Choisne, J.

Strimpakos, N., see Dimitriadis, Z.

Sudoyo, H., see Haryono, I. R.

Takemura, H., see Nomura, K.

20 (2017) 1750001

20 (2017) 1772001

20 (2017) 1750009

20 (2017) 1750013

20 (2017) 1772001

20 (2017) 1750022

20 (2017) 1750008

20 (2017) 1750003

20 (2017) 1750019

20 (2017) 1750012

20 (2017) 1750011 
Tanaka, Y., see Nomura, K.

20 (2017) 1750011

Tayyem, M., Elhadid, F., Haddad, R., Samarah, O., Ananzah, M., Ahmad, M. and Hamdan, M., Perioperative Use of Pregabalin in Total Knee Arthroplasty: MetaAnalysis of Randomized Controlled Trials

20 (2017) 1750009

Tedla, J. S., see Midde, A. K.

20 (2017) 1750020

Toby, E. B., see Chappell, I.

20 (2017) 1750006

Toliopoulos, I. K., see Oikonomidis, S. A.

20 (2017) 1750008

Travert, C., see Choisne, J.

20 (2017) 1750003

Tulaar, A., see Haryono, I. R.

20 (2017) 1750012

Ughareja, P., see Salunke, A. A.

20 (2017) 1772001

Valiadis, J.-M., see Choisne, J.

20 (2017) 1750003

Van den Heever, D., see Garikayi, T.

20 (2017) 1750015

Verginadis, I. I., see Oikonomidis, S. A.

20 (2017) 1750008

Yamauchi, K., see Oishi, Y.

20 (2017) 1750007

Yonezawa, T., see Nomura, K.

20 (2017) 1750011

Yoon, M.-G., see Moon, M.-S.

20 (2017) 1750002

Yoon, M.-G., see Moon, M.-S.

20 (2017) 1750010

Zulfeequer, C. P., see Sahasrabudhe, A.

20 (2017) 1750023 